\title{
Finite Element Modeling of Magneto-superelastic Behavior of Ferromagnetic Shape Memory Alloy Helical Springs
}

\author{
Jong-Bin Lee ${ }^{1}$, Yutaka Toi ${ }^{2}$, and Minoru Taya ${ }^{3}$ \\ ${ }^{1}$ Graduate School, University of Tokyo, Komaba, Meguro-ku, \\ Tokyo 153-8505, Japan \\ jongbin@iis.u-tokyo.ac.jp \\ ${ }^{2}$ Institute of Industrial Science, University of Tokyo, Komaba, Meguro-ku, \\ Tokyo 153-8505, Japan \\ toi@iis.u-tokyo.ac.jp \\ ${ }^{3}$ Center for Intelligent Materials and Systems, University of Washington, Seattle, \\ WA 98195-2600, USA \\ tayam@u.washington.edu
}

\begin{abstract}
The previous study by thefor the finite element analysis of superelastic, large deformation behaviors of one-dimensional shape memory alloy (SMA) devices is extended to the magneto-superelastic analysis of ferromagnetic shape memory alloy (FSMA) devices. The commercial code ANSYS/ Emag for magnetic analysis is combined with the SMA superelastic analysis program developed by the authors. The numerical results for a SMA (NiTi) beam are compared with the experimental results to illustrate the reliability of the superelastic analysis program. The magneto-superelastic analysis of FSMA helical springs is conducted to show the validity of the developed analysis system.
\end{abstract}

\section{Introduction}

Ferromagnetic shape memory alloy (abbreviated as FSMA) is under development and is expected as a new material of shape memory alloy (abbreviated as SMA) actuators. The conventional thermoelastic SMA exhibits large strain and also bears large stress. However their actuation speed is usually slow, since the actuation speed is limited by the heating/cooling rates of the SMA. On the other hand, FSMA is driven by applied magnetic field and hence can provide very fast actuation speed with reasonably large strain and stress capability. For this reason, FSMA actuator is attracting attention as a new device, for example, for a morphing aircraft, which is capable of changing the shape of wings like a bird to reduce drag and improve fuel consumption.

It is expected that a computational tool will be used more widely in the design of FSMA-based actuators. However, the computational method has not yet been established for the magneto-superelastic analysis of FSMA helical springs, which are standard components of FSMA actuators. In the present study, the finite element analysis is conducted for the magneto-superelastic behavior of FSMA helical springs. The commercial code ANSYS/ Emag and the superelastic analysis program developed by the authors [1] are used for the magnetic analysis and the superelastic analysis, respectively. In the program, Brinson's one-dimensional constitutive modeling [2], 
which is relatively simple and phenomenological, is extended to consider the asymmetric tensile and compressive behavior as well as the torsional behavior. The incremental finite element formulation by the total Lagrangian approach is employed for the layered linear Timoshenko beam element equipped with the extended Brinson's constitutive equation. The present method is simple and efficient as it employs the beam element. The calculated results for the superelastic deformation analysis of a SMA (Ni-Ti-10\%Cu) beam subjected to 4-point bending as well as the magnetosuperelastic analysis of a FSMA helical spring subjected to magnetic forces generated by the hybrid magnet composed of a permanent and electro magnet are compared with the experimental results to show the validity of the present method.

\section{Magneto-superelastic Analysis of FSMA Helical Springs}

\subsection{Magnetic Analysis}

The commercial code ANSYS/Emag is used for the magnetic analysis. The method of difference scalar potential is employed with eight-noded hexahedron elements for magnetic solids and four-noded tetrahedron elements (SOLID96) for space. Sixnoded trigonal prism elements (INFINI11) are used as infinite elements. SOURCE36 is used to model electric source. In ANSYS, magnetic forces are calculated in the air elements adjacent to the body of interest. Sufficiently fine and non-distorted mesh should be used to obtain accurate magnetic forces, however, it requires considerable meshing effort and computing time. Then the helical spring is simplified as an assembly of circular rings as shown in Fig. 1 with a rectangular cross-section as shown in Fig. 2 in order to avoid the difficulty in meshing for the three-dimensional space of complicated shape surrounding the surface of the helical spring.
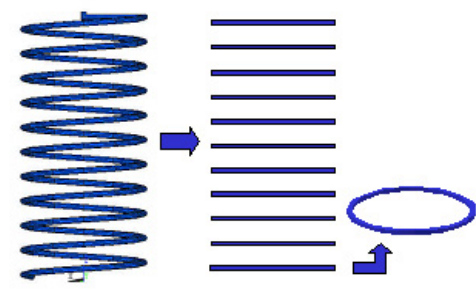

Fig. 1. Idealization of helical spring

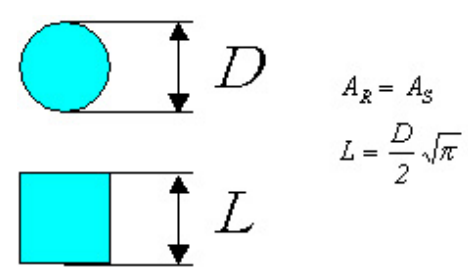

Fig. 2. Cross-sections

Fig. 3 shows the correspondence between the models for the magnetic and the structural (superelastic) analysis (see also Fig. 9). In the superelastic analysis, the first turn of the helical spring is subjected to the calculated magnetic force on the first ring as shown in Fig. 3(a). The n-th turn of the helical spring is subjected to the calculated magnetic force on the $n$-th ring under the condition that all rings above the n-th ring are stacked on the yolk of the electromagnet as shown in Fig. 3(b). The stacking effect in the FSMA spring is important as the magnetic flux transmits the spring wire in self-contact. The calculated magnetic forces here give the upper bound values as they are calculated on the assumption that all rings above the ring of interest are stacked. Then, the calculated magnetic forces are reasonable under the strong magnetic flux, while this assumption can make some error under the weak magnetic flux. 


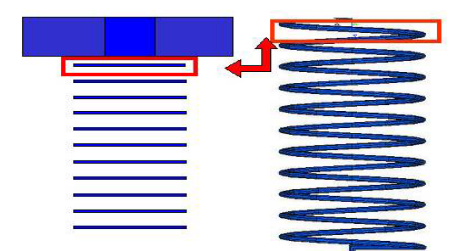

magnetic analysis structural analysis (a) magnetic force for the first turn

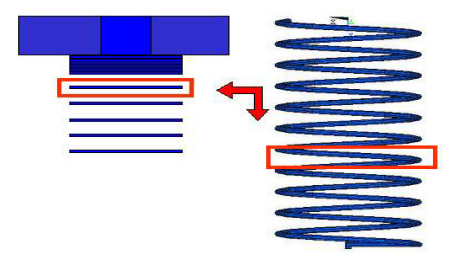

magnetic analysis structural analysis

(b) magnetic force for the $\mathrm{n}$-th turn

Fig. 3 Modeling for magnetic analysis and structural (superelastic) analysis

\subsection{Superelastic Deformation Analysis}

\subsubsection{Constitutive Equation for Shape Memory Alloy}

The mechanical property of SMA is schematically shown in Fig. 4 [2]. Fig. 4(a) and Fig. 4(b) are the critical transformation stress versus and temperature and the superelastic stress-strain behavior respectively, in which the following symbols are used: $T$; temperature, $\sigma$; stress, $\varepsilon$; strain, $\sigma_{f}^{c r}$ and $\sigma_{s}^{c r}$; critical finishing and starting stress of martensite transformation, $C_{M}$ and $C_{A}$; slope for the relation between critical transformation stress and temperature, $M_{f}$ and $M_{s}$; critical finishing and starting temperature of martensite transformation, $A_{s}$ and $A_{f}$; critical starting and finishing temperature of austenite transformation. The loading and unloading at the temperature higher than $A_{f}$ cause the superelastic stress-strain behavior as shown in Fig. 4(b).

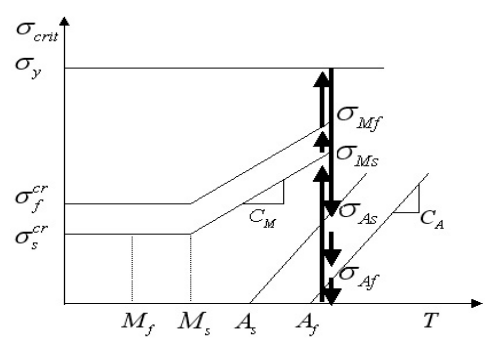

(a) transformation stress vs. temperature

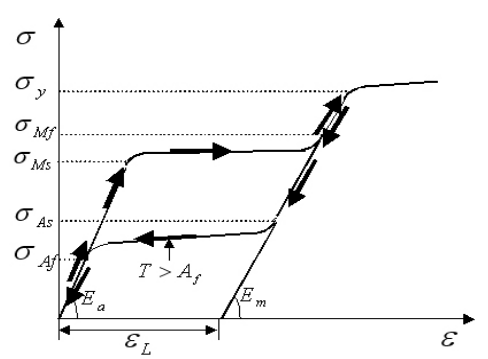

(b) superelastic stress-strain behavior

Fig. 4 Mechanical property of shape memory alloy

The one-dimensional stress-strain relation is generally written as

$$
\sigma-\sigma_{0}=E\left(\varepsilon-\varepsilon_{0}\right)+\Omega\left(\xi_{S}-\xi_{S 0}\right)+\theta\left(T-T_{0}\right)
$$

where $E$; Young's modulus, $\Omega$; transformation coefficient, $\xi_{S}$; stress-induced martensite volume fraction, $\theta$; thermal elastic coefficient, $T$; temperature. The subscript ' 0 ' indicates the initial values. $\Omega$ is expressed as

$$
\Omega=-\varepsilon_{L} E
$$


where $\varepsilon_{L}$ is the maximum residual strain. Young's modulus $E$ is a function of the martensite volume fraction $\xi$, which is given by

$$
E=E_{a}+\xi\left(E_{m}-E_{a}\right)
$$

where $E_{m}$ and $E_{a}$ are Young's modulus of austenite phase and martensite phase respectively. The total martensite volume fraction $\xi$ is expressed as

$$
\xi=\xi_{S}+\xi_{T}
$$

where $\xi_{T}$ is the temperature-induced martensite volume fraction. $\xi, \xi_{S}$ and $\xi_{T}$ are functions of the temperature $T$ and the stress $\sigma$. To consider the difference between tensile and compressive behavior, von Mises equivalent stress $\sigma_{e}$ in the evolution equations of $\xi, \xi_{S}$ and $\xi_{T}$ is replaced with Drucker-Prager equivalent stress $\sigma_{e}^{D P}$ [3] defined as

$$
\sigma_{e}^{D P}=\sigma_{e}+3 \beta p
$$

where $\beta$ is the material parameter and $p$ is the hydrostatic pressure given by

$$
p=\frac{1}{3}\left(\sigma_{x}+\sigma_{y}+\sigma_{z}\right)
$$

In one-dimensional case, the equivalent stress in eq. (5) is expressed as

$$
\sigma^{D P}=|\sigma|+\beta \sigma
$$

Substituting eq. (7) into the evolution equations of $\xi, \xi_{S}$ and $\xi_{T}$ given by Brinson [2], the evolution equations for the transformation to martensite phase and austenite phase are expressed as follows:

(i) transformation to martensite phase

$$
\begin{gathered}
T>M_{s} \text { and } \sigma_{s}^{c r}(1+\beta)+C_{M}(1+\beta)\left(T-M_{s}\right)<\sigma^{D P}<\sigma_{f}^{c r}(1+\beta)+C_{M}(1+\beta)\left(T-M_{s}\right): \\
\xi_{S}=\frac{1-\xi_{S 0}}{2} \cos \left\{\frac{\pi}{\sigma_{s}^{c r}(1+\beta)-\sigma_{f}^{c r}(1+\beta)}\left[\sigma^{D P}-\sigma_{f}^{c r}(1+\beta)-C_{M}(1+\beta)\left(T-M_{s}\right)\right]\right\}+\frac{1+\xi_{S 0}}{2} \\
\xi_{T}=\xi_{T 0}-\frac{\xi_{T 0}}{1-\xi_{S 0}}\left(\xi_{S}-\xi_{S 0}\right)
\end{gathered}
$$

$T<M_{s}$ and $\sigma_{s}^{c r}(1+\beta)<\sigma^{D P}<\sigma_{f}^{c r}(1+\beta)$ :

$$
\begin{gathered}
\xi_{S}=\frac{1-\xi_{S 0}}{2} \cos \left\{\frac{\pi}{\sigma_{s}^{c r}(1+\beta)-\sigma_{f}^{c r}(1+\beta)}\left[\sigma^{D P}-\sigma_{f}^{c r}(1+\beta)\right]\right\}+\frac{1+\xi_{S 0}}{2} \\
\xi_{T}=\xi_{T 0}-\frac{\xi_{T 0}}{1-\xi_{S 0}}\left(\xi_{S}-\xi_{S 0}\right)+\Delta_{T \xi}
\end{gathered}
$$

where $M_{f}<T<M_{s}$ and $T<T_{0}$ :

$$
\Delta_{T \xi}=\frac{1-\xi_{T 0}}{2}\left\{\cos \left[a_{M}\left(T-M_{f}\right)\right]+1\right\}
$$


Otherwise

$$
\Delta_{T \xi}=0
$$

(ii) transformation to austenite phase

$$
\begin{gathered}
T>A_{s} \text { and } C_{A}(1+\beta)\left(T-A_{f}\right)<f<C_{A}(1+\beta)\left(T-A_{s}\right): \\
\xi=\frac{\xi_{0}}{2}\left\{\cos \left[a_{A}\left(T-A_{s}-\frac{f}{C_{A}(1+\beta)}\right)\right]+1\right\} \\
\xi_{S}=\xi_{S 0}-\frac{\xi_{S 0}}{\xi_{0}}\left(\xi_{0}-\xi\right) \\
\xi_{T}=\xi_{T 0}-\frac{\xi_{T 0}}{\xi_{0}}\left(\xi_{0}-\xi\right)
\end{gathered}
$$

where $a_{M}$ and $a_{A}$ are given by the following equations:

$$
a_{M}=\frac{\pi}{M_{s}-M_{f}}, \quad a_{A}=\frac{\pi}{A_{f}-A_{s}}
$$

It is assumed for simplicity that the superelastic shear deformation behavior is qualitatively similar to the normal deformation behavior and both are independent with each other [4]. In the evolution equations for the martensite volume fractions due to the shear stress, $\xi_{\tau}, \xi_{S \tau}$ and $\xi_{T \tau}$ are used for the shear deformation. $\sqrt{3}|\tau|$ is employed instead of $\sigma^{D P}$ in eq. (7). The shear stress-shear strain relation is expressed by the following equation:

$$
\tau-\tau_{0}=G\left(\gamma-\gamma_{0}\right)+\Omega_{\tau}\left(\xi_{S \tau}-\xi_{S \tau 0}\right)
$$

where $G$; shear modulus, $\Omega_{\tau}$; shear transformation constant, $\xi_{S \tau}$; shear stressinduced martensite volume fraction, $T$; temperature. The subscript ' 0 ' indicates the initial value. $\Omega_{\tau}$ is expressed as follows:

$$
\Omega_{\tau}=-\gamma_{L} G_{\tau}
$$

where $\gamma_{L}$ is the maximum residual strain. The shear modulus $G$ is a function of the martensite volume fraction $\xi_{\tau}$, which is given by

$$
G_{\tau}=G_{a}+\xi_{\tau}\left(G_{m}-G_{a}\right)
$$

where $G_{m}$ and $G_{a}$ are the elastic shear modulus of martensite phase and austenite phase respectively. The total martensite volume fraction $\xi_{\tau}$ is expressed as

$$
\xi_{\tau}=\xi_{S \tau}+\xi_{T \tau}
$$

where $\xi_{T \tau}$ is the temperature-induced martensite volume fraction. $\xi_{\tau}, \xi_{S \tau}$ and $\xi_{T \tau}$ are functions of the temperature $T$ and the shear stress $\tau$.

$\sqrt{3}|\tau|$ is used as the equivalent stress to express the evolution equations of the martensite volume fractions due to shear, which are given by the following replacements in eqs. (8) to (17): 


$$
\begin{aligned}
& f \rightarrow \sqrt{3}|\tau|, \quad \beta=0, \quad \xi \rightarrow \xi_{\tau}, \quad \xi_{0} \rightarrow \xi_{\tau 0}, \quad \xi_{S} \rightarrow \xi_{S \tau}, \quad \xi_{S 0} \rightarrow \xi_{S \tau 0}, \\
& \xi_{T} \rightarrow \xi_{T \tau}, \quad \xi_{T 0} \rightarrow \xi_{T \tau 0}, \quad \Delta_{T \xi} \rightarrow \Delta_{T \tau \xi}
\end{aligned}
$$

\subsubsection{Finite Element Formulation}

\section{Incremental Constitutive Equation}

The layered linear Timoshenko beam element [5] as shown in Fig. 5 is used in the finite element analysis of SMA helical springs. The superelastic behavior is assumed for the normal stress $(\sigma)$-normal strain $(\varepsilon)$ behavior associated with the axial and bending deformation as well as the shear stress $(\tau)$-shear strain $(\gamma)$ behavior associated with the torsional deformation.

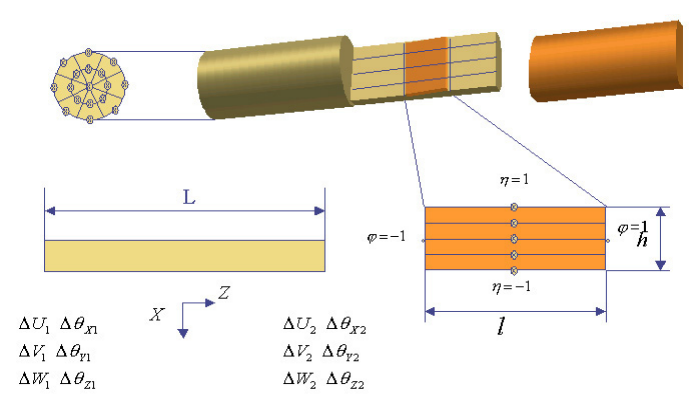

Fig. 5 Layered Timoshenko beam element

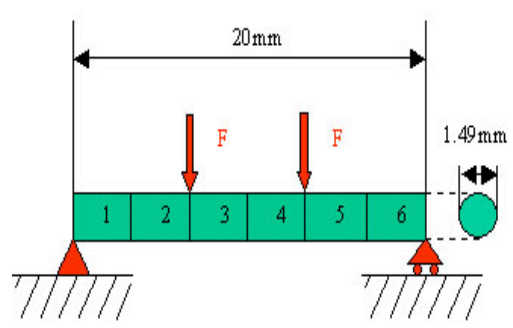

Fig. $6 \mathrm{Ni}-\mathrm{Ti}-10 \% \mathrm{Cu}$ alloy beam

The shear deformation associated with the bending deformation is assumed to be elastic and the shear strain energy due to bending is treated as a penalty term because the effect of bending is smaller than torsion in helical springs. The incremental stressstrain relation for the analysis of helical springs is written in the following form:

$$
\{\Delta \sigma\}=\left[D_{s e}\right]\left(\{\Delta \varepsilon\}-\left\{\Delta \varepsilon_{s e}\right\}\right)
$$

where

$$
\{\Delta \sigma\}=\left\{\begin{array}{c}
\Delta \sigma \\
\Delta \tau_{x z} \\
\Delta \tau_{y z} \\
\Delta \tau
\end{array}\right\}, \quad\left[D_{s e}\right]=\left[\begin{array}{cccc}
E_{s e} & 0 & 0 & 0 \\
0 & G & 0 & 0 \\
0 & 0 & G & 0 \\
0 & 0 & 0 & G_{s e}
\end{array}\right], \quad\{\Delta \varepsilon\}=\left\{\begin{array}{c}
\Delta \varepsilon \\
\Delta \gamma_{x z} \\
\Delta \gamma_{y z} \\
\Delta \gamma
\end{array}\right\},\left\{\Delta \varepsilon_{s e}\right\}=\left\{\begin{array}{c}
\Delta \varepsilon_{s e} \\
0 \\
0 \\
\Delta \gamma_{s e}
\end{array}\right\}
$$

in which $\tau_{x z}$ and $\tau_{y z}\left(\gamma_{x z}\right.$ and $\left.\gamma_{y z}\right)$ are the shear stresses (strains) due to bending. The final form of eq. (23) is given by Toi et al. [1].

\section{Incremental Stiffness Equation}

The effect of large deformation is taken into account by using the incremental theory by the total Lagrangian approach in which the nonlinear terms with respect to the displacement in the axial direction are neglected [1]. The following element stiffness equation in an incremental form is obtained by the finite element formulation based on the total Lagrangian approach $[5,6]$ : 


$$
\left(\left[k_{0}\right]+\left[k_{L}\right]+\left[k_{G}\right]\right)\{\Delta u\}=\{\Delta f\}+\left\{f_{R}\right\}+\int_{V_{e}}[\bar{B}]^{T}[D]\left\{\Delta \varepsilon_{\theta}\right\} d V^{(0)}
$$

where

$$
\begin{gathered}
{\left[k_{0}\right]=\int_{V_{e}}\left[B_{0}\right]^{T}[D]\left[B_{0}\right] d V^{(0)}} \\
{\left[k_{L}\right]=\int_{V_{e}}\left(\left[B_{0}\right]^{T}[D]\left[B_{L}\right]+\left[B_{L}\right]^{T}[D]\left[B_{0}\right]+\left[B_{L}\right]^{T}[D]\left[B_{L}\right]\right) d V^{(0)}} \\
{\left[k_{G}\right]=\int_{V_{e}}[G]^{T}[S][G] d V^{(0)}}
\end{gathered}
$$

Table 1. Material constants of $\mathrm{Ni}-\mathrm{Ti}-10 \% \mathrm{Cu}$ alloy

\begin{tabular}{cccc}
\hline $\begin{array}{c}\text { Elastic moduli } \\
\text { and } \beta\end{array}$ & $\begin{array}{c}\text { Transformation } \\
\text { temperatures }\end{array}$ & $\begin{array}{c}\text { Transformation } \\
\text { constants }\end{array}$ & $\begin{array}{c}\text { Maximum } \\
\text { residual strain }\end{array}$ \\
\hline$E_{a}=60 \times 10^{3} \mathrm{MPa}$ & $M_{f}=T_{0}-72.5^{\circ} \mathrm{C}$ & $C_{M}=8.0 \mathrm{MPa} /{ }^{\circ} \mathrm{C}$ & \\
$E_{m}=20 \times 10^{3} \mathrm{MPa}$ & $M_{s}=T_{0}-52.5^{\circ} \mathrm{C}$ & $C_{A}=13.8 \mathrm{MPa} /{ }^{\circ} \mathrm{C}$ & \\
$\theta=0.55 \mathrm{MPa} /{ }^{\circ} \mathrm{C}$ & $A_{s}=T_{0}-21.7^{\circ} \mathrm{C}$ & $\sigma_{s}{ }^{c r}=100 \mathrm{MPa}$ & $\varepsilon_{L}=0.067$ \\
$\beta=0.15$ & $A_{f}=T_{0}-14.5^{\circ} \mathrm{C}$ & $\sigma_{f}{ }^{c r}=180 \mathrm{MPa}$ & \\
& & & \\
\hline
\end{tabular}

The following symbols are used: $\left[k_{0}\right]$; the incremental stiffness matrix, $\left[k_{L}\right]$; the initial displacement matrix, $\left[k_{G}\right]$; the initial stress matrix, $\{\Delta f\}$; the external force increment vector, $\left\{f_{R}\right\}$; the unbalanced force vector, $\left[D_{s e}\right]$; the superelastic stressstrain matrix, $\left\{\Delta \varepsilon_{s e}\right\}$; the superelastic initial strain vector, $[G]$; the gradient matrix, $[S]$; the initial stress matrix, $V_{e}$; the element volume.

\section{Numerical Examples}

\subsection{Superelastic Analysis of SMA Beam}

The numerical analysis for a SMA ( $\mathrm{Ni}-\mathrm{Ti}-10 \% \mathrm{Cu}$ ) beam subjected to 4 -point bending has been conducted to illustrate the validity of the superelastic analysis program. Fig. 6 shows a simply supported Ni-Ti-10\% Cu alloy beam subjected to 4-point bending. The material constants of Ni-Ti-10\% Cu alloy have been determined, based on the material test results [3]. Fig. 7 shows the comparison between the experimental and the assumed stress-strain curves for $\mathrm{Ni}-\mathrm{Ti}-10 \% \mathrm{Cu}$ alloy. The assumed material constants are shown in Table 1. Fig. 8 shows the calculated load-central deflection curves assuming the symmetric $(\beta=0)$ and asymmetric $(\beta=0.15)$ tensile and compressive stress-strain behaviors. The calculated load-displacement curve by using Drucker-Prager equivalent stress $(\beta=0.15)$ has agreed much better with the experimental curve given by Auricchio and Taylor [3] than the result with von Mises equivalent stress $(\beta=0)$. 


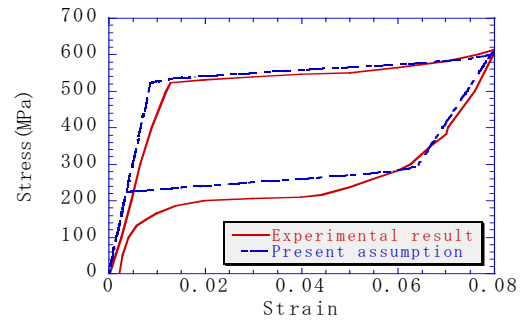

Fig. 7. Stress-strain relations

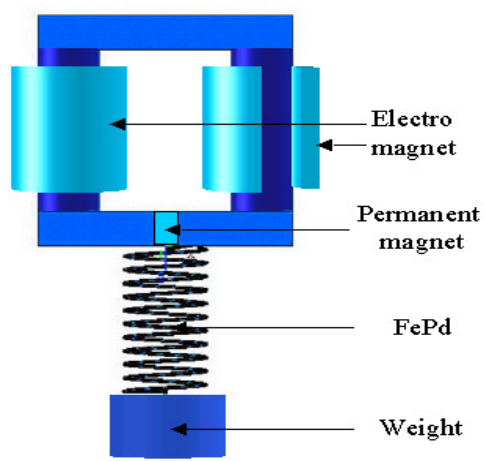

Fig. 9. FePd helical spring on hybrid magnet

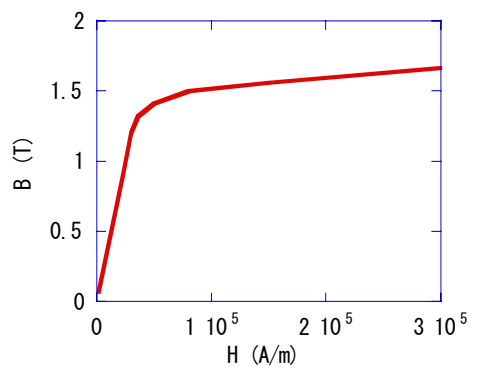

(a) B-H curve for FePd

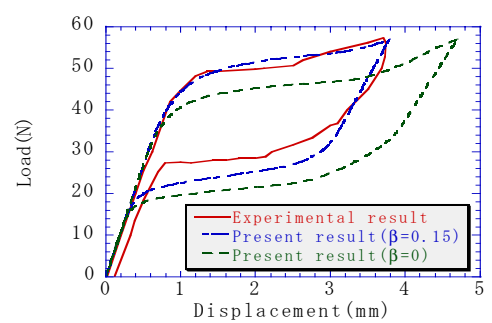

Fig. 8. Load-displacement curves

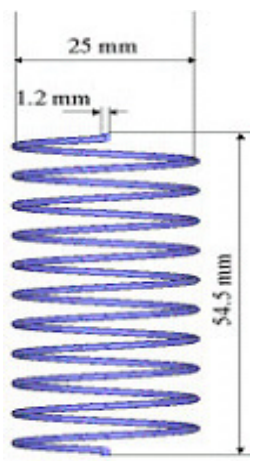

Fig. 10. Dimensions of FePd spring

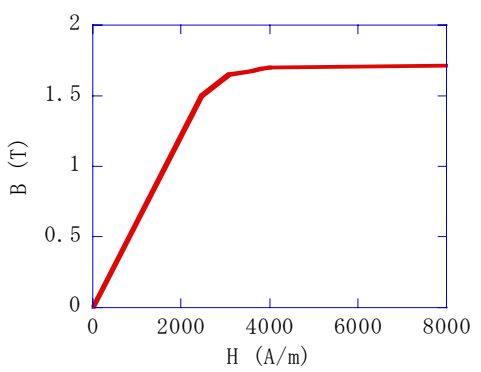

(b) B-H curve for yolk

Fig. 11. Permeability of FePd and yolk

\subsection{Magneto-superelastic Analysis of FSMA Helical Spring}

Fig. 9 shows a FePd helical spring with a weight subjected to a magnetic force by the permanent magnet (Niodume35, relative permeability : 1.17, coercive force : 835563 $\mathrm{A} / \mathrm{m}$ ) and the electro-magnet (798 turns, 0 1.0A). Fig. 10 shows the dimensions of the helical spring to be analyzed.

Fig. 11 shows B-H curves for FePd and yolk. The material constants of FePd have been determined, based on the tensile test result. The assumed stress-strain curve is 
compared with the test result in Fig. 12. The assumed material constants are shown in Table 2. Fig. 13 shows the calculated magnetic forces at various current levels. Fig. 14 shows the calculated current-displacement curves for FSMA helical springs with and without a weight $(0.49 \mathrm{~N})$. The calculated displacements have corresponded well with the experimental results given by the CIMS at the University of Washington as an upper bound solution (see the subsection 2.1).

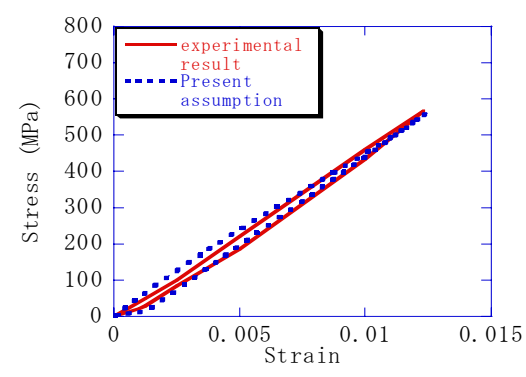

Fig. 12. Stress-strain curves for FePd

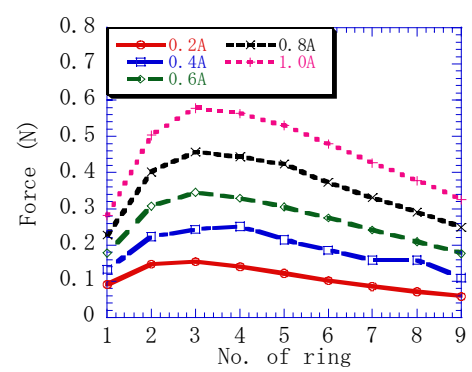

Fig. 13. Magnetic force distribution

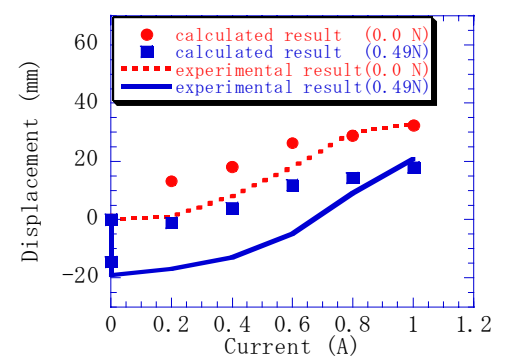

Fig. 14. Current-displacement curves

Table 2. Material constants of FePd

\begin{tabular}{clc}
\hline $\begin{array}{c}\text { Elastic moduli }(\mathrm{MPa}) \\
\text { and } \beta\end{array}$ & \multicolumn{1}{c}{$\begin{array}{c}\text { Transformation } \\
\text { constants }(\mathrm{MPa})\end{array}$} & $\begin{array}{c}\text { Maximum residual } \\
\text { strains }\end{array}$ \\
\hline & $\sigma_{M s}=\sigma_{s}^{c r}+C_{M}\left(T-M_{s}\right)=20$ & \\
$E_{m}=49000$ & $\sigma_{M f}=\sigma_{f}^{c r}+C_{M}\left(T-M_{s}\right)=560$ & $\varepsilon_{L}=0.001$ \\
$E_{a}=53000$ & $\sigma_{A s}=C_{A}\left(T-A_{s}\right)=18$ & $\gamma_{L}=0.001$ \\
$\beta=0.15$ & $\sigma_{A f}=C_{A}\left(T-A_{f}\right)=2$ & \\
& & \\
\hline
\end{tabular}

\section{Concluding Remarks}

The finite element analysis has been conducted for the magneto-superelastic behavior of FSMA helical springs in the present study, in which Brinson's constitutive modeling has been extended to consider the asymmetric tensile and compressive behavior 
as well as the torsional behavior. The numerical result for a SMA beam subjected to 4-point bending has agreed well with the experimental result. The magnetosuperelastic behavior of a FSMA helical spring has been analyzed. The calculated current-upper bound displacement curves have corresponded well with the experimental results.

\section{References}

1. Toi Y, Lee JB, Taya M, Finite element analysis of superelastic, large deformation behavior of shape memory alloy helical springs, Computers \& Structures, 82(20/21), 2004, 1685-1693.

2. Brinson LC, One-dimensional constitutive behavior of shape memory alloy: thermomechanical derivation with non-constant material functions and redefined martensite internal variable, Journal of Intelligent Material Systems and Structures, 4, 1993, 229-242.

3. Auricchio F, Taylor RL, Shape memory alloy: modeling and numerical simulations of the finite-strain superelastic behavior, Comput. Methods Appl. Mech. Engng., 143, 1997, 175194.

4. Sun QP, Li ZQ, Phase transformation in superelastic NiTi polycrystalline micro-tubes under tension and torsion: from localization to homogeneous deformation, Int. J. Solids Structures 2002, 39, 3797-3809.

5. Bathe KJ, Finite Element Procedures, Prentice Hall, 1996.

6. Washizu K, Variational methods in elasticity and plasticity, 3rd Ed., Pergamon Press, 1982. 